\title{
Chewing the Fat: Is Autophagy a Potential Therapeutic Target for Atherosclerosis and Other Diseases of Sterol Metabolism?
}

\author{
W. Gray (Jay) Jerome ${ }^{1,2}$, Courtney Netherland-Van Dyke ${ }^{1}$, Carrie Romer ${ }^{1}$ \\ ${ }^{1}$ Department of Pathology, Microbiology and Immunology, Vanderbilt University, Nashville, TN, USA \\ ${ }^{2}$ Department of Cancer Biology, Vanderbilt University, Nashville, TN, USA
}

Atherosclerosis is a disease of medium to large arteries where the artery wall thickens because of the accumulation of lipid, cells and debris. A key feature is the presence of macrophages that are filled with cholesterol. This cholesterol is primarily derived from the endocytosis of modified LDL (mLDL) particles. In LDL particles the cholesterol occurs as cholesteryl esters (CE); a cholesterol molecule esterified to a fatty acid (FA). In early phases of the disease, the LDL cholesteryl ester is digested in the lysosome and the resultant free cholesterol (FC) is transported out of the lysosome. Excess sterol is reesterified to a fatty acid in the cytoplasm and is stored in lipid droplets along with triglycerides (TG) in the cytoplasm. The cells become so full of lipid droplets that they appear foamy and so are called foam cells. The cytoplasmic storage of lipid is well documented in early atherosclerosis. However, in late stages of the disease, sterol accumulation in the lysosomes disrupts normal lysosome function and both free and esterified cholesterol accumulates creating large, lipid-engorged lysosomes. These dysfunctional lysosomes disrupt normal macrophage function and exacerbate the atherosclerotic disease.

Autophagy is a degradative process whereby cellular structures are targeted to lysosomes for degradation. As such, it helps maintain homeostasis by ridding the cell of worn out components and toxic compounds. The process is regulated by a series of autophagy-related genes. There is evidence that autophagy participates in atherosclerosis but how it does so is not clear[1]. Recently, it was reported that autophagy can target lipids (cholesteryl esters and triglycerides) in cytoplasmic lipid droplets to lysosomes for degradation (Figure 1). Moreover, in an animal model of early atherosclerosis, it has been shown that inhibiting autophagy in macrophage foam cells can increase the amount of atherosclerosis[2]. These studies conclude that autophagy delivers cholesteryl esters from lipid droplets back to lysosomes for conversion to unesterified cholesterol so that it can be removed from cells in a process called efflux.

We hypothesized that although autophagy may help clear cholesterol from cells in early lesions, in late-stage clinically important lesions (where lysosomes are dysfunctional) delivery of cholesteryl esters to lysosomes would worsen the lysosome dysfunction. To test this hypothesis, we employed a cell culture model of late stage atherosclerosis. Macrophages in culture were engorged with cholesterol by incubation with aggregates of LDL (agLDL) to levels equivalent to that seen in lesion foam cells. These cholesterol-stuffed cells exhibited sterol engorged lysosomes like the lysosomes of atherosclerosis (Figure 2A). Looking at specific marker proteins that are increased when autophage is stimulated (Atg5, p62, LC3) we found that there is constitutively active autophagy in macrophages and that the levels do not change with sterol accumulation. However, one marker, LC3-II, did increase when the cells were engorged with sterol. Unlike the early stage markers we examined, LC3II is a late stage autophagy marker which is delivered to lysosomes with autophagy vesicles and degraded. Since other markers for autophagy did not increase, we conclude that LC3-II was delivered to lysosomes but built up because it was not degraded. These studies indicate that autophagy was present in foam cells but did not prove that lipid was targeted to lysosomes. To look at lipid targeting, we examined whether LC3-II and adipophilin (a lipid droplet associated protein) were delivered to lysosomes. This was done by fluorescence microscopy colocalization studies (Figures 2B and 2C). We found that as sterol levels increased in cells, there was an increase in colocalization of both LC3-II and adipophilin with the lysosome marker LAMP-1. Thus, it appears that increases in sterol in foam cells does not stimulate autophagy but the already active autophagy pathway targets lipid from the cytoplasmic droplets and delivers them to the lysosome. The LC3-II and adipophilin data, however, suggest that the delivered lipids and proteins are not digested but act to increase even further the buildup of material in lysosomes. To confirm this, we isolated lysosomes from cells and analyzed their content. In the late stage foam cells, the lysosomes contained significant amounts of sterol and LC3-II and adipophilin. As a final test 
of our hypothesis that this targeting of cytoplasmic cholesteryl ester back to lysosomes did not aid in the clearance of cholesterol, we measured the lysosome $\mathrm{pH}$ using the fluorescent dye, lysosensor yellow/blue. This dye fluoresces yellow in an acidic environment but becomes blue shifted as $\mathrm{pH}$ increases. We counted the number of active and inactive lysosomes in cells. We considered a lysosome with $\mathrm{pH}<5$ as active and those with $\mathrm{pH}>5$ as inactive. Sterol treatment produced a 50\% decrease in active lysosomes after 6 days of sterol loading and this was the time that LC3-II and adipophilin accumulation was maximal.

Our studies indicate that autophagy is a major route for delivery of cholesterol to lysosomes but that it would only be a viable therapeutic target if the therapy was combined with one that restored function to dysfunctional lysosomes.

[1] X Liao et al, Cell Metab 15:545-453 (2012)

[2] M Ouimet et al, Cell Metabolism 13:655-667 (2011)

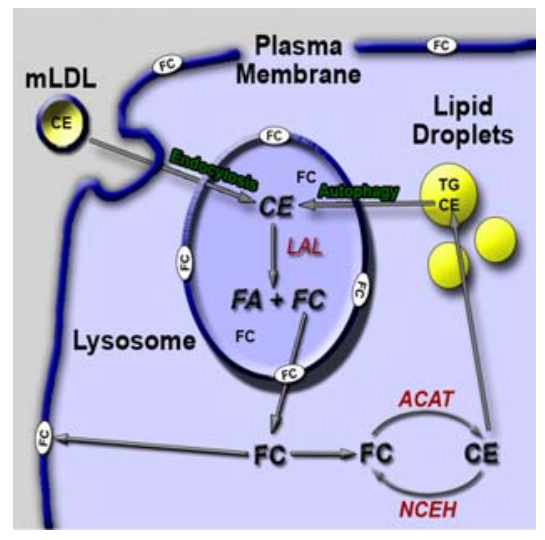

Figure 1: Delivery of CE to lysosomes in macrophages. Two delivery routes are available. Exogenous CEs in lipoproteins are delivered by endocytosis. Endogenous CEs in lipid droplets can be delivered by autophagy. CE cannot be cleared from lysosomes, it must be digested by lysosomal acid lipase (LAL) to FC in order to be cleared.
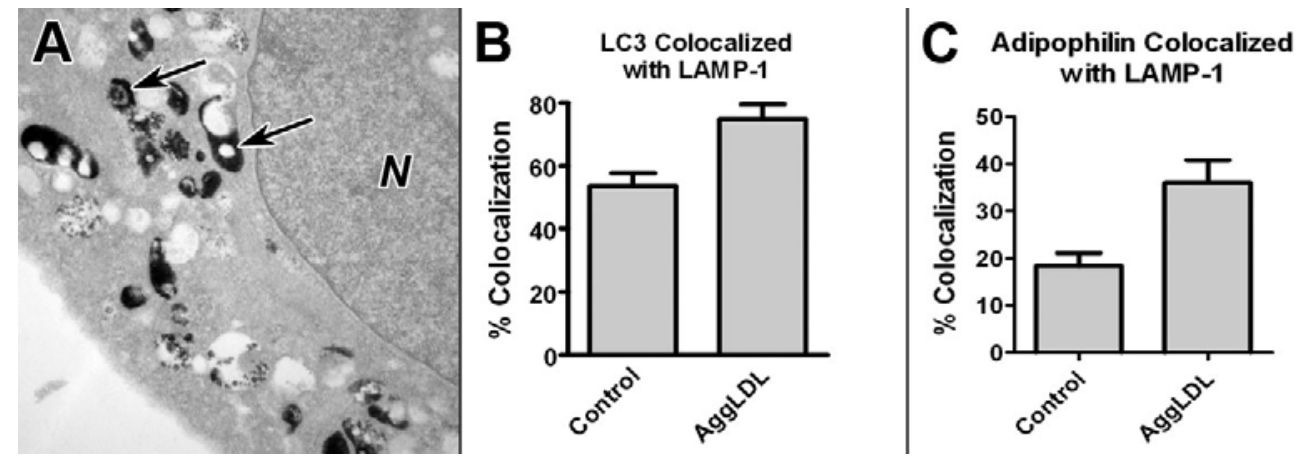

Figure 2: Macrophage lysosomal CE metabolism. A) TEM of late stage foam cell in culture showing $\mathrm{CE}$ and other lipids accumulating in lysosomes. Cells were reacted to demonstrate lysosome hydrolases (black precipitate, arrows), $\mathrm{N}=$ nucleus, magnification $=1,850 \mathrm{X}$. B) Percent of LC3 that is found within lysosomes (LAMP-1 positive). C) Percent of adipophilin found within lysosomes (LAMP-1 positive). 